\title{
Renal disease more prevalent and problematic for Aboriginal peoples
}

A boriginal peoples in Canada are far more likely to require treatment for kidney failure and end-stage renal disease than other Canadians, but don't fare as well following dialysis and have lower rates of kidney transplantation, according to a new report from the Canadian Institute for Health Information (CIHI).

Patients from the Aboriginal population who receive dialysis have a lower 5-year survival rate than the general population ( $40 \%$ vs. $45 \%$ ). Those requiring a kidney transplant also trail the rest of Canada, by a wide margin, in actually receiving one (27\% versus $42 \%$ ).

In all, $2 \%$ of patients with chronic kidney disease develop end-stage renal disease, which has no cure. Aboriginal peoples, however, are nearly three times as likely as non-Aboriginal patients to receive treatment for end-stage renal disease. The main treatment options are dialysis or a kidney transplant.

The higher prevalence of end-stage renal disease among Aboriginal peoples is associated with higher rates of obesity and diabetes. Compared to other Canadians with the condition, patients from the Aboriginal community are more likely to be obese (40\% vs. $27 \%$ ) and to have diabetes (49\% vs. 27\%), according to the report, End-Stage Renal Disease Among Aboriginal Peoples in Canada: Treatment and Outcomes.

One of the primary reasons Aboriginal patients with end-stage renal disease fare poorly following dialysis is that they often live far away from treatment centres. About one in five has to travel more than $250 \mathrm{~km}$. And since treatment is sometimes required multiple times a week, for several hours at a time, this can affect employment, as well as make it difficult to have the support of family or friends.

There are also several barriers to organ transplantation in Aboriginal communities, including a lack of education resources about the procedure and cultural beliefs that frown upon the practice.

Diabetes too has a negative impact on treatment options, particularly for transplants, says Sushma Mathur, manager of the Canadian Population Health Initiative at CIHI.

Patients with diabetes are more likely to experience postoperative complica- tions, as are donors who have diabetes. "There are a whole range of emotional and psychological factors that would also need to be considered," says Mathur.

Proposed interventions to improve the kidney health of Aboriginal peoples include bringing more health services to the community, training health care workers on culturally appropriate practices and erasing geographical barriers through technology such as telehealth.

"We also found that community involvement in the planning of prevention and treatment services was shown to improve outcomes," says Mathur.

In the future, CIHI hopes to have access to data on specific populations - First Nations, Métis and Inuit within the Aboriginal community.

"We know that there are clinical and treatment differences for these specific populations," says Mathur. "In the future, it would be great if our data systems could be a bit more sophisticated so that we can actually report on each of the groups within the Aboriginal population in Canada." — Roger Collier, CMAJ

CMAJ 2013. DOI:10.1503/cmaj.109-4412

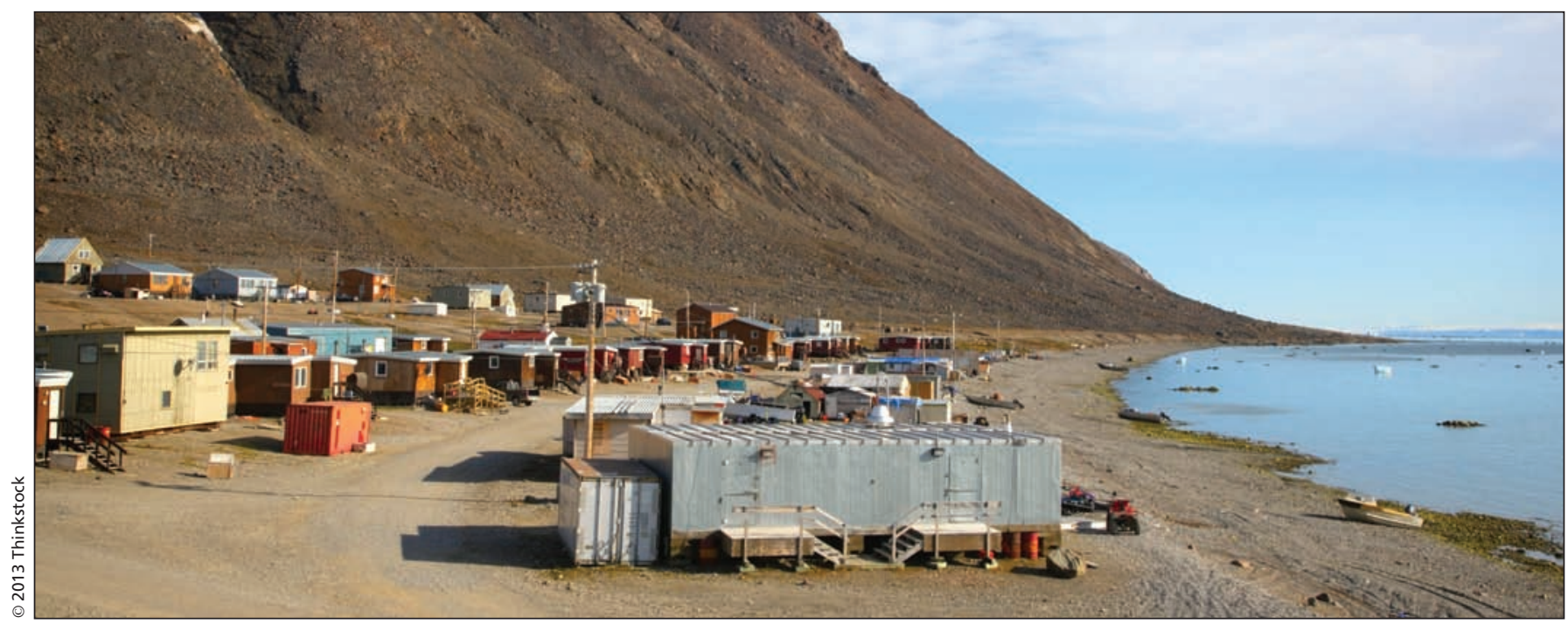

Aboriginal peoples from remote communities — such as Grise Fiord, Nunavut - tend to fare poorly following dialysis because they often live far away from treatment centres. 